\title{
Desarrollo y salud: la emergencia de un nuevo paradigma
}

\author{
Jesús María Sánchez-Ordóñez \\ Magíster en Salud Pública. Trabajador Social \\ Universidad del Valle. Cali, Colombia \\ https://orcid.org/0000-0002-5633-665X・jesus.sanchez@correounivalle.edu.co
}

Resumen

Sobre el desarrollo y la salud como nociones de primer orden en todas las sociedades, existe una amplia gama de definiciones y teorías desde casi todas las ciencias y disciplinas en las que se dan por sentadas las implicaciones que mantienen entre sí. Ambos conceptos resurgieron después de la Segunda guerra mundial, impulsados por la ONU y la OMS respectivamente. Ante la ausencia de estudios que demuestren con evidencia esas implicaciones, en este artículo se presentan elementos del estudio comparativo realizado con el fin de analizar la relación existente entre las nociones de desarrollo y salud en el mundo actual, mediante algunos elementos que permitan reflexionar sobre el diseño e implementación de intervenciones tanto en el ámbito del desarrollo como en el de la salud.

El análisis demostró que la idea hegemónica de que el desarrollo corresponde al crecimiento económico no ha posibilitado el mejoramiento de las condiciones de salud y bienestar en las sociedades, lo cual se evidencia en las asimetrías existentes entre los países del continente europeo con respecto a los continentes americano y asiático. En consecuencia, se requiere con urgencia replantear los marcos de referencia con mayores niveles de desarrollo, con el fin de incorporar nociones más amplias que reconozcan la complejidad de los entornos y garanticen la sostenibilidad en el planeta y el disfrute de la vida.

Palabras clave: Desarrollo; Salud; Desarrollo sostenible; Desarrollo humano; Desarrollo económico.

Recibido: 17/02/2020 |Aprobado: 05/10/2020 |Publicado: 01/01/2021

(c) (1) (3) Esta obra está bajo una Licencia Creative Commons Atribución-NoComercialCompartirIgual 4.0 Internacional.

Financiación o proveniencia del artículo: artículo derivado del proyecto de tesis doctoral "Universidad saludable, sus componentes, enfoques y alcances como aporte al desarrollo local" (en construcción) del Programa Doctorado en desarrollo local y cooperación internacional. Universidad Jaume I de Castellón (España).

¿Cómo citar este artículo? / How to quote this article?

Sánchez-Ordóñez, J. M. (2021). Desarrollo y salud: la emergencia de un nuevo paradigma. Prospectiva. Revista de Trabajo Social e intervención social, (31), 413-436. doi: $10.25100 /$ prts.v0i31.8934. 
Sánchez-Ordóñez

\title{
Development and Health: The Emergence of a New Paradigm
}

\begin{abstract}
Regarding development and health as first-order notions in all societies, there is a wide range of definitions and theories from almost all sciences and disciplines, in which the implications they hold for each other are taken for granted. Both concepts resurfaced after the Second World War, promoted by the UN and the WHO respectively. In the absence of studies that demonstrate these implications with evidence, this article presents elements of a comparative study carried out to analyse the relationship between the two notions in today's world. The study contains some elements that allow us to reflect on the design and implementation of interventions in both fields.

The analysis showed that the hegemonic idea that development corresponds to economic growth has not made it possible to improve the conditions of health and wellbeing in societies, a fact that is evidenced in the asymmetries existing between the countries of the European continent with respect to those of the American and Asian continents. Consequently, it is urgently required to rethink the reference frameworks with higher levels of development, in order to incorporate broader notions that recognize the complexity of environments and guarantee sustainability on the planet and the enjoyment of life.

Keywords: Development; Health; Sustainable development; Human development; Economic development.

Sumario: 1. Introducción, 2. Metodología, 3. Hallazgos, 3.1. Descripción de la literatura seleccionada, 3.2. Comparación de países según indicadores de desarrollo y salud, 3.3. Aproximaciones a la evolución de los paradigmas de desarrollo, 3.4. La salud y el desarrollo, 3.5. Desafíos para el desarrollo y la salud en el siglo XXI, 4. Conclusiones, 5. Referencias bibliográficas.
\end{abstract}




\section{Introducción}

Los cambios que han surgido en el mundo después de la Segunda guerra mundial, han puesto dentro de las prioridades globales dos asuntos que, a primera vista, son muy diferentes pero que, como demuestran las evidencias, tienden a confundirse: el desarrollo y la salud. El desarrollo, entendido como el propósito fijo para todos los Estados, es concebido desde el crecimiento económico y considerado requisito indispensable para el bienestar de las sociedades. Por su parte, la salud es definida desde entonces por la Organización Mundial de la Salud (OMS), (1946) como "el completo estado de bienestar físico, mental y social, y no solo ausencia de enfermedad".

Estudios como los de Brenner, quien ha dedicado su interés a teorizar sobre la relación entre tasas de desempleo, niveles de crecimiento económico y tasas de morbilidad física y mental, han sido soporte para el diseño de políticas sociales y económicas en Estados Unidos, pues se ha insistido en que en las épocas de recesión, con el aumento de tasas de desempleo, aumentan los niveles de enfermedad mental y suicidio, entre otras, con sus cargas globales tanto para el Estado como para los individuos y la sociedad en general. De otro lado, el crecimiento económico acumulado se asocia con la disminución de mortalidad, en el caso de Estados Unidos, con base en estudios de series entre el año 1901 al 2000 (Brenner, 2005).

Las teorías de Brenner han sido debatidas e incluso controvertidas por otros autores que afirman que, por el contrario, las tasas de enfermedad mental y física se elevan en los periodos de alto crecimiento económico. Para Ruhm, por ejemplo, habría una fuerte evidencia de que la salud mejoraría así no creciera la economía temporalmente (García, 2017). Los críticos de Brenner controvierten incluso los fundamentos epistemológicos, teóricos y metodológicos de sus estudios; no obstante, han sido referencia para la toma de decisiones políticas en Estados Unidos y en otros países, incluso europeos.

A pesar de que el debate persistente sobre la relación entre desarrollo y salud es de larga data y el acervo documental es amplio, paradójicamente no aparecen en la literatura científica estudios que recojan las publicaciones dispersas existentes y generen evidencia contundente sobre la asociación de variables de salud y los indicadores de desarrollo en el mundo (Higuita-Gutiérrez y Cardona-Arias, 2018).

Estudios como el de Tafani, Chiesa, Caminati y Gaspio (2015), que por asociatividad toma variables como el daño ambiental y la desigualdad social como elementos relevantes del desarrollo y su impacto sobre la calidad de vida, la salud y sus dimensiones, tratan de dar cuenta de esta relación. Por lo tanto, es relevante aportar elementos que permitan responder estos interrogantes: ¿Qué relación hay entre las nociones y modelos de desarrollo contemporáneos con la salud integral de las poblaciones? y ¿Cómo es posible mejorar los 
Sánchez-Ordóñez

niveles de salud en el mundo? Esto considerando que diariamente en todos los entornos geopolíticos, territoriales, institucionales y sociales se toman decisiones con el fin de obtener mejores niveles de desarrollo, algo a lo que los ciudadanos no se oponen por ser deseable a pesar de que el anhelo de vivir mejor, disfrutar de la vida en todos los ámbitos, alargar la felicidad y no enfermarse cada vez más parece una utopía para la gran mayoría de las poblaciones.

La OMS y la Organización de Naciones Unidas (ONU) han venido buscando estrategias para frenar los impactos negativos que ponen en cuestión la vida en el planeta. De ahí que, en las últimas tres décadas, han reconocido, mediante la evidencia, el efecto de los Determinantes Sociales sobre la Salud (DSS) de las personas, lo cual ha impulsado, desde los diferentes escenarios multinacionales y poli temáticos, la adopción de decisiones y acciones políticas para contrarrestar estas problemáticas.

Asumir los DSS es distanciarse de las reformas neoliberales y aceptar su fracaso e incapacidad para solucionar complejos problemas actuales de la salud. Este enfoque se diferencia sustancialmente de la perspectiva de factores de riesgo y se ubica en la comprensión de las inequidades entre grupos sociales. Inequidades entre las que sobresalen la distribución desigual de bienes sociales como: ingreso, riqueza, empleo digno y con estabilidad, alimentación y hábitos saludables, acceso a servicios como la salud, la educación y la recreación entre otros (Álvarez-Castaño, 2014).

Los informes sobre desarrollo humano, en particular el de 2019, acogiendo esta misma línea analiza las desigualdades en el mundo desde un nuevo marco asumiéndolas más allá del ingreso, de los promedios y mirando más hacia el futuro, pues aspectos como la crisis climática, los adelantos tecnológicos en el campo de la inteligencia artificial, entre otros, afectan principalmente a los pobres, amenazando incluso a colectivos enteros de personas y países (Programa de las Naciones Unidas para el Desarrollo, 2019).

El reconocer la influencia de los DSS en la calidad de vida de las comunidades e individuos ha llevado a impulsar intervenciones en todos los entornos, y a crear estrategias como municipios saludables, organizaciones saludables, escuelas saludables, entre otras.

Tanto el desarrollo como la salud son dos dimensiones que avanzan en paralelo con sus propios corpus teóricos, epistemológicos y metodológicos; sin embargo, ambos conceptos están implicados desde su génesis tras el nuevo orden mundial establecido en la segunda posguerra. Es evidente que los niveles de desarrollo afectan las condiciones de salud y que el grado de salud de una población determina su desarrollo. Pero por alguna razón no evidente, tanto los economistas como los salubristas persisten en sus desarrollos científicos separadamente, asumiendo cada concepto como un fin en sí mismo o, en el mejor de los casos, cada concepto se asume como medio para el otro. 
Sánchez-Ordóñez

Siguiendo el rastro de estas nociones de desarrollo con el fin de entender un poco más los paradigmas contemporáneos y reconociendo la magnitud y diversidad de estas nociones, en el presente estudio se han clasificado en tres grandes grupos o categorías de la siguiente manera:

Desarrollo 1.0: este primer momento en las teorías y modelos de desarrollo corresponde a la idea de desarrollo como crecimiento económico per sé, en el que se obliga a los Estados a priorizar las políticas de crecimiento como única vía para el bienestar y el desarrollo social de sus ciudadanos.

Desarrollo 2.0: corresponde a las teorías y modelos que anteponen el bienestar y desarrollo social al crecimiento económico. En este grupo emergen conceptos como capital humano, capital social, desarrollo humano, gerencia social, innovación social e incluso desarrollo sostenible, solo que siguen supeditados a la idea de desarrollo como aumento del nivel de recursos materiales.

Desarrollo 3.0: este también se podría denominar como el de las teorías alternativas del desarrollo, en el que la riqueza material y económica dejan de ser un fin, son solo uno de los recursos requeridos para el buen vivir, el desarrollo humano desde las capacidades y libertades, dando preponderancia a la cultura tal como lo propone Sen $(2000,2004)$, la sostenibilidad ecológica como medio de supervivencia. El fin del desarrollo es el ser humano y sus entornos.

La salud en este grupo es vista desde una perspectiva holista en la que se rebasa la noción biomédica, incorporando a las dimensiones definidas por la OMS, las dimensiones espiritual y ambiental, de tal forma que la salud no se reduce a la esfera biológica/física y no se considera como un fin en sí misma.

A esta clasificación de nociones o paradigmas del desarrollo subyace la idea de evolución y complejidad de las mismas, no se trata de una mera evolución lineal en el tiempo, sino que tal como se catalogan los desarrollos del software en el campo de las tecnologías de información, se da una estructuración más amplia y acabada entre la versión posterior (3.0) y la anterior (2.0), o de la (2.0) con respecto a (1.0) dejando espacio incluso para que los modelos sigan evolucionando en el futuro.

En este orden, se realizó la presente revisión sistemática, cuyo objetivo principal fue analizar la relación existente entre modelos de desarrollo y la salud en el mundo, para aportar elementos de reflexión para el debate y para enfrentar los desafíos actuales en la intervención de los entornos humanos. 


\section{Metodología}

Para dar respuesta al objetivo mencionado, se asumió la perspectiva interpretativa siguiendo la estrategia PICO (Mamédio, Roberto \& Nobre, 2007) según la cual el ámbito/problema abordado es la relación directa entre desarrollo y salud, para identificar si con mayores niveles de desarrollo mejoran las dimensiones de la salud de las poblaciones e individuos, con base en reflexiones y estudios recogidos en los textos científicos.

Se realizó una revisión sistemática basada en el modelo PRISMA (Moher, Liberati, Tetzlaff, Altmane y Grupo PRISMA, 2014) por medio de las siguientes bases de datos: Dialnet, Scielo, Fuente Académica Premier, Complementary Index, DOAJ, Business Source Complete, Academic Search Complete, Gale Academic Onefile, Google Scholar, Jstor Journals, Medline, Redalyc, Web of Science, Pubmed, Oxford Journals, Ebsco. En todas estas se definió como patrón de búsqueda la palabra clave "Desarrollo y Salud" restringida a publicaciones académicas arbitradas. De manera retrospectiva, se seleccionó un rango temporal desde 1998 a 2019. La bitácora de búsqueda aplicada fue: TI desarrollo y salud; OR AB desarrollo y salud; AND AB teorías del desarrollo; AND AB modelos de desarrollo; NOT $\mathrm{AB}$ enfermedades.

Inicialmente, se encontraron 845 documentos de los cuales se descartaron 179 por no estar en idiomas inglés, español o portugués. De los 666 artículos filtrados se descartaron 630 por repetición y por no mostrar en el título ninguna relación con las categorías de estudio o por tratar áreas distintas. Los 36 seleccionados se incluyeron en una matriz de EXCEL de elaboración propia, en la que se clasificaron por año, objetivos, metodología, resultados, conclusiones, palabras clave, tipo de estudio y base de datos. Finalmente, se seleccionaron 29 artículos que cumplían todos los criterios.

Tras saturar el campo, se realizó una búsqueda complementaria con la intención de aumentar el alcance del estudio; para ello, se seleccionaron 8 documentos como bases de datos de la ONU, la OMS y del Banco mundial, así como dos libros de texto.

Con los documentos seleccionados de manera definitiva se realizó el análisis cualitativo, apoyado en una tabla comparativa con criterios cuantitativos agrupados en seis variables: Producto Interno Bruto (PIB), Producto Interno Bruto Per Cápita (PPC), Esperanza de Vida al Nacer (EVN), tasas de fecundidad, población menor de 15 años y mayor de 65 años. Se tomó como muestra a conveniencia 23 países con relevancia por sus niveles de desarrollo y salud de cuatro continentes (Europa, América, Asia y Oceanía). 


\section{Hallazgos}

\subsection{Descripción de la literatura seleccionada}

La búsqueda de documentos sobre desarrollo y salud desde 1998 a noviembre de 2019, se fijó por el interés de encontrar literatura desde la década de los años 90, que fue una época poscrisis global en la que se implementó una amplia gama de reformas estatales que involucraron las nociones de desarrollo y salud. Se realizó el primer filtrado descartando documentos que no estuviesen disponibles para consulta; se seleccionaron artículos tanto de investigación como de reflexión y revisión, pues la naturaleza del objeto de estudio es amplia y compleja; además que, como se mencionó, ha sido poco abordada desde la relación entre los dos conceptos (desarrollo y salud), se eliminaron los estudios duplicados, y todos aquellos que hacían referencia a uno de los dos conceptos y que tenían escasa relación con el objetivo de estudio.

El tipo de estudio para aquellas publicaciones que no lo tenían se dedujo del resumen y/o de la introducción. El proceso de revisión seguido según el protocolo PRISMA aparece en la Figura 1.

Figura 1. Resultados de la búsqueda según método PRISMA.
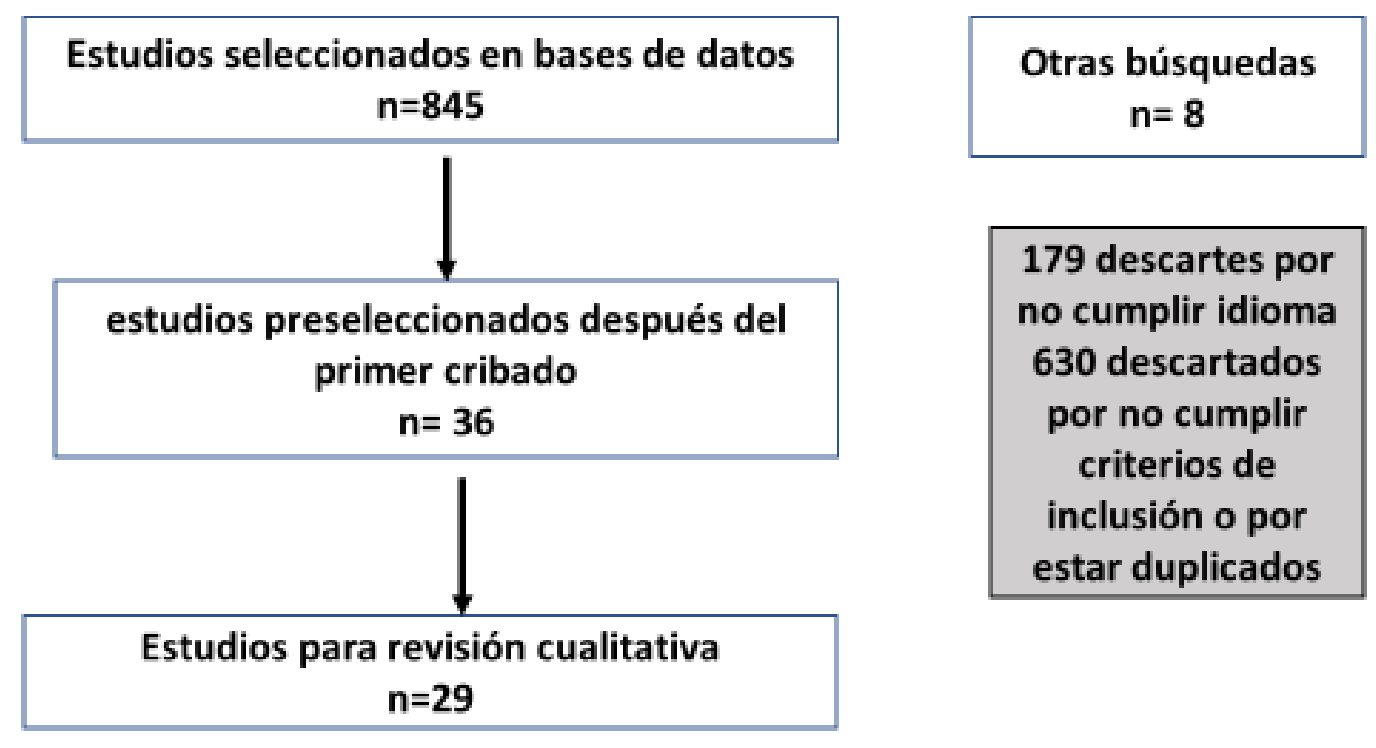

\section{9 descartes por no cumplir idioma 630 descartados por no cumplir criterios de inclusión o por estar duplicados}

Fuente: elaboración propia.

En la Tabla 1 se observa que, de los 29 artículos, el $48 \%$ se escribieron en los últimos cinco años del periodo establecido para el análisis. Dentro de los artículos clasificados en la noción de desarrollo 1.0 encontramos que se centran en el eje del crecimiento económico, los daños a la salud, al ambiente y a la sociedad, así como aspectos asociados al desarrollo 
Sánchez-Ordóñez

territorial y la cooperación internacional. Los que corresponden al desarrollo 2.0 tienen como eje temático principalmente las desigualdades, la equidad, las inequidades y la salud como condición para el desarrollo justo y la sostenibilidad ambiental. Los estudios enmarcados en el desarrollo 3.0 abordan temáticas más amplias, entre ellas el desarrollo humano desde la perspectiva más antropológica, axiológica, espiritual, ambiental y política.

Tabla 1. Total de estudios seleccionados en la revisión

\begin{tabular}{|c|c|c|c|}
\hline $\begin{array}{c}\text { Año } \\
\text { publicación }\end{array}$ & Autor & $\begin{array}{l}\text { Modelo de } \\
\text { desarrollo }\end{array}$ & Eje central \\
\hline 1999 & (Agudelo, 1999) & 3.0 & $\begin{array}{l}\text { Desarrollo humano } \\
\text { sostenible }\end{array}$ \\
\hline 2005 & (Brenner, 2005) & 1.0 & Crecimiento \\
\hline 2006 & (Cejudo-Córdoba, 2006) & 3.0 & $\begin{array}{l}\text { Desarrollo humano } \\
\text { promoción de la libertad }\end{array}$ \\
\hline 2007 & (Lustig, 2007) & 2.0 & Desigualdades sociales \\
\hline 2008 & (Casella, 2008) & 1.0 & Crítica al crecimiento \\
\hline 2009 & $\begin{array}{l}\text { (Mora-Alvarado y Portuguez- } \\
\text { Barquero, 2009) }\end{array}$ & 1.0 & Factores ambientales \\
\hline 2010 & (De Souza-Silva, 2010) & 1.0 & Crítica al crecimiento \\
\hline 2011 & (Muñoz-Muñoz, 2011) & 2.0 & Equidad social \\
\hline 2012 & (Pratt \& Loff, 2012) & 2.0 & $\begin{array}{l}\text { Investigación para reducir } \\
\text { inequidades }\end{array}$ \\
\hline 2012 & $\begin{array}{l}\text { (Grabois-Gadelha, Costa \& } \\
\text { Maldonado, 2012) }\end{array}$ & 2.0 & Innovación en salud \\
\hline 2012 & $\begin{array}{l}\text { (Ríos-Osorio, Salas-Zapata \& } \\
\text { Ortiz-Lobato, 2012) }\end{array}$ & 3.0 & $\begin{array}{l}\text { Salud por fuera del marco } \\
\text { institucional de desarrollo } \\
\text { sostenible }\end{array}$ \\
\hline 2013 & (Manet, 2013) & 1.0 & Desarrollo territorial \\
\hline 2013 & (Salaverry-García, 2013) & 2.0 & Equidad en salud \\
\hline 2014 & (Sager, 2014) & 3.0 & Sostenibilidad ambiental \\
\hline 2015 & (Gamarra-Pérez, 2015) & 3.0 & $\begin{array}{l}\text { Integración hombre, valores, } \\
\text { espiritualidad }\end{array}$ \\
\hline 2015 & $\begin{array}{c}\text { (Rubio-Guerrero y Fierro-Celis, } \\
\text { 2015) }\end{array}$ & 1.0 & Sustentabilidad ambiental \\
\hline 2015 & $\begin{array}{l}\text { (Siero, Rodríguez y Hernández, } \\
\text { 2015) }\end{array}$ & 1.0 & Cooperación internacional \\
\hline 2015 & (Tafani et al., 2015) & 1.0 & $\begin{array}{l}\text { Más desarrollo mayor daño } \\
\text { ambiental y daño a la salud }\end{array}$ \\
\hline 2015 & (Tellería, 2015) & 3.0 & Poder en vez de desarrollo \\
\hline
\end{tabular}


Sánchez-Ordóñez

2016

(Forouzanfar et al., 2016)

Ruiz-Lurduy, Rocha-Buelvas,

2016 Pérez-Hernández y Córdoba-Sánchez, 2016)

2016

(Escobar y Esteva, 2017)

2017

(Feigin et al., 2017)

(García, 2017)

(García-Rodríguez, García-

2017

Fariñas, Priego-Hernández y Martíez-

Pérez, 2017)

2017

2017

2018

2019
(Arias, 2017)

(Sarmiento-Suárez, 2017)

(Higuita-Gutiérrez y Cardona-

Arias, 2018)

(St'ahel, 2019)
1.0

3.0
Más desarrollo mayor daño ambiental y daño a la salud

Salud intercultural

Posdesarrollo "vivir más allá del desarrollo"

Más desarrollo aumenta enfermedades neuronales y mortalidad

Menos crecimiento mayor desempleo más enfermedad

Más salud más desarrollo

Sostenibilidad justa

Más salud mental más desarrollo

Más desarrollo humano, más salud, mayor desarrollo

Cambio de modelo de desarrollo para enfrentar el cambio climático

Fuente: elaboración propia.

El $62 \%$ de los artículos como se aprecia en la Tabla 2 corresponden a trabajos de reflexión, el 27,5 a investigación y el 10,3 a revisiones. De estos estudios, el 38\% se clasificó dentro del modelo de desarrollo 1.0, el 31\% en el modelo de desarrollo 2.0 y, el $31 \%$ en el modelo 3.0. Llama la atención que de los artículos de investigación la mayoría se ubican en el modelo 1.0 y el resto en el 3.0, ninguno aparece en el modelo 2.0, lo que refuerza la idea del gran interés global en el modelo 1.0.

Cabe anotar también que dentro de los artículos de investigación se recogen estudios que analizan la asociación entre algunas variables del desarrollo como crecimiento económico, desempleo, desarrollo tecnológico, nivel de riqueza y variables del ámbito de la salud, esperanza de vida, tasas de fecundidad, tasas de suicidio, y múltiples indicadores de enfermedad física y mental en el mundo (Brenner, 2005; GBD 2015 Neurological Disorders Collaborator Group, 2017; GBD 2015 Risk Factors Collaborators, 2016; Mora-Alvarado y Portuguez-Barquero, 2009). 
Sánchez-Ordóñez

Tabla 2. Estudios según modelo de desarrollo y tipo

\begin{tabular}{cccccc}
\hline & Desarrollo 1.0 & Desarrollo 2.0 & Desarrollo 3.0 & Total & Porcentaje \\
\hline Investigación & 5 & & 3 & 8 & $27,5 \%$ \\
Reflexión & 5 & 7 & 6 & 18 & $62,2 \%$ \\
Revisión & 1 & 2 & & 3 & $10,3 \%$ \\
Total & 11 & 9 & 9 & 29 & $100 \%$ \\
\hline Porcentaje & $38 \%$ & $31 \%$ & $31 \%$ & $100 \%$ \\
\hline \multicolumn{7}{c}{ Fuente: elaboración propia }
\end{tabular}

\subsection{Comparación de países según indicadores de desarrollo y salud}

En la Tabla 3 es posible apreciar que los países que tienen la esperanza de vida más alta son Japón, Singapur, Australia, España, Italia, Suiza y Noruega; en América, los países de mayor esperanza de vida son Canadá, Costa Rica, Cuba y Chile.

En Europa se observa correspondencia entre nivel de riqueza económica y esperanza de vida al nacer; en Italia y España, que tienen mayor PIB, la esperanza de vida es de 83 años. En general, los países de la muestra tienen una EVN alta, aunque no tengan iguales niveles de riqueza.

En el continente americano se observa asimetría, dado que el país de mayor riqueza no es el que cuenta con una esperanza de vida más alta y, por el contrario, países como Canadá, Costa Rica, Cuba y Chile tienen un índice más alto. Estados Unidos, que es el país de mayor PIB, tiene una esperanza de vida de 79,6 años, inferior incluso a los países de Europa incluidos en la muestra que tienen PIB muy inferiores.

En Asia sobresale Japón que es el país de mayor PIB y tiene la esperanza de vida más alta entre todos los países de la muestra; Israel y Singapur, con PIB inferiores, tienen índices similares a los países europeos. En Oceanía, el país de mayor PIB y mayor esperanza de vida es Australia.

Las tasas de fecundidad global más bajas corresponden en su orden a Singapur, España, Italia, Japón, Luxemburgo y Suiza.

En general, los países europeos y asiáticos de la muestra, a excepción de Israel y de Oceanía, presentan bajas tasas de fecundidad. En América, la tasa más baja la tienen Canadá y Cuba.

En cuanto a la composición demográfica, los países europeos de la muestra son los que concentran la mayor tasa de población mayor de 65 años, siendo Italia, Suecia y España los que tienen la tasa más alta. En Asia, es muy significativo el caso de Japón con la tasa más alta de población mayor de 65 años de toda la muestra. Los países americanos aún 
Sánchez-Ordóñez

muestran tasas de población mayor de 65 años inferiores a Europa, teniendo los valores más altos Canadá, Estados Unidos y Cuba.

Israel, Panamá, México y Argentina son los países con tasas más altas de población menor de 15 años. Por el contrario, Japón, Italia, España, Suiza y Catar son los que presentan las menores tasas de población con edad inferior a 15 años. Israel y Oceanía, presentan bajas tasas de fecundidad. En América, la tasa más baja la tienen Canadá y Cuba.

Tabla 3. Comparación de países según indicadores de desarrollo y salud.

\begin{tabular}{|c|c|c|c|c|c|c|c|c|}
\hline \multirow[t]{2}{*}{ Continente } & \multirow{2}{*}{$\begin{array}{l}\text { Ranking } \\
\text { según PIB } \\
\text { per cápita }\end{array}$} & \multirow[t]{2}{*}{ Países } & \multirow{2}{*}{$\begin{array}{c}\text { PIB } \\
\text { (billones } \\
\text { de USD) }\end{array}$} & \multirow{2}{*}{$\begin{array}{l}\text { PIB per } \\
\text { cápita } \\
\text { USD }\end{array}$} & \multicolumn{2}{|c|}{ población } & \multirow{2}{*}{$\begin{array}{c}\text { Tasa de } \\
\text { Fecundidad }\end{array}$} & \multirow{2}{*}{$\begin{array}{c}\text { Esperanza } \\
\text { de vida al } \\
\text { nacer }\end{array}$} \\
\hline & & & & & $\begin{array}{l}<15 \\
\text { años }\end{array}$ & $\begin{array}{l}>65 \\
\text { años }\end{array}$ & & \\
\hline & & 2017 & & & & & 2018 & \\
\hline \multirow{9}{*}{ América } & 8 & Estados Unidos & 19.485 & 59.927 & 19 & 16 & 1,9 & 79,7 \\
\hline & 11 & Canadá & 1.646 & 45.069 & 16 & 17 & 1,6 & 82,7 \\
\hline & 22 & México & 1.158 & 9.281 & 26 & 7 & 2,1 & 77,5 \\
\hline & 20 & Argentina & 642 & 14.591 & 25 & 11 & 2,3 & 76,9 \\
\hline & 19 & Chile & 277 & 15.037 & 20 & 11 & 1,8 & 79,9 \\
\hline & 23 & Cuba & 96 & 8.541 & 16 & 15 & 1,7 & 80,1 \\
\hline & 18 & Panamá & 62 & 15.166 & 27 & 8 & 2,5 & 78,4 \\
\hline & 21 & Costa Rica & 58 & 11.752 & 21 & 10 & 1,8 & 80,2 \\
\hline & 17 & Uruguay & 56 & 16.437 & 21 & 15 & 2 & 77,8 \\
\hline \multirow{8}{*}{ Europa } & 14 & Italia & 1.946 & 32.155 & 14 & 23 & 1,4 & 83 \\
\hline & 16 & España & 1.314 & 28.208 & 15 & 19 & 1,3 & 83 \\
\hline & 3 & Suiza & 678 & 80.333 & 15 & 18 & 1,5 & 83 \\
\hline & 10 & Suecia & 535 & 53.253 & 18 & 20 & 1,9 & 82 \\
\hline & 4 & Noruega & 399 & 75.704 & 18 & 17 & 1,7 & 83 \\
\hline & 5 & Irlanda & 334 & 75.648 & 22 & 14 & 1,9 & 82 \\
\hline & 2 & Luxemburgo & 62 & 104.498 & 16 & 14 & 1,5 & 82 \\
\hline & 6 & Islandia & 24 & 71.314 & 20 & 15 & 1,8 & 82 \\
\hline \multirow{5}{*}{ Asia } & 13 & Japón & 4.859 & 38.332 & 13 & 27 & 1,4 & 84 \\
\hline & 12 & Israel & 353 & 40.543 & 28 & 12 & 3,1 & 82 \\
\hline & 7 & Singapur & 338 & 60.297 & 15 & 13 & 1,2 & 83 \\
\hline & 1 & Catar & 166 & 128.378 & 14 & 1 & 1,9 & 78 \\
\hline & 15 & Brunéi & 12 & 28.572 & 23 & 5 & 1,9 & 77 \\
\hline Oceanía & 9 & Australia & 1.330 & 54.093 & 19 & 16 & 1,8 & 83 \\
\hline
\end{tabular}

Fuente: elaboración propia con datos de la Organización Panamericana de la Salud (OPS), 2018; base de datos generada del portal del Banco Mundial el 19 de noviembre de 20191

1 Los datos sobre PIB per cápita se refieren mayoritariamente al año 2017, según diversas fuentes: World Development Indicators Database ("Base de datos de indicadores de desarrollo mundial"), Banco Mundial (BM), Base de datos generada del portal del Banco mundial el 19 de noviembre de 2019. 


\subsection{Aproximaciones a la evolución de los paradigmas de desarrollo}

Como lo muestra la revisión de estudios sobre la relación salud-desarrollo. en los últimos años se han publicado principalmente reflexiones en torno las nociones sobre el desarrollo, así como algunos resultados de investigación que asocian variables de salud y de desarrollo con el fin de aportar elementos para la profundización o transformación de las ideas que se tienen respecto a estos constructos.

Salud y desarrollo son conceptos multidimensionales que encierran gran complejidad debido a que se soportan en ideologías, creencias, valores y sobre todo en intereses de grupos específicos. No se cuenta con definiciones universales, por lo que cada autor se aproxima desde su línea investigativa a su comprensión. No obstante, la revisión evidenció el marcado desbalance sobre el modelo 1.0, que a pesar de estar asociado a los momentos iniciales de las teorías del desarrollo como crecimiento económico, persiste con total vigencia en la actualidad, superando las nociones que incorporan otras dimensiones como la social, la antropológica, la filosófica, entre otras.

Es por esto que entrar en los debates históricos sobre las nociones, modelos o paradigmas de desarrollo y su implicación en el bienestar y la salud de las personas, nos remite obligatoriamente al marco de la segunda pre y posguerra mundial en el siglo XX, dada la relevancia de lo que esto ha significado para todos los países del mundo.

No significa esto que antes de ese momento histórico se carecía de tales nociones, pues lo primero que hay que develar es la naturaleza del concepto de desarrollo como constructo humano mediado por los contextos socioculturales e históricos en los que se produce. De ahí que, a lo largo de la historia de la humanidad, las ideas sobre evolución, crecimiento, avance, cambio, transformación, entre otras similares, han marcado las acciones de las diferentes sociedades y Estados.

Dicho esto, se comienza por reconocer la cantidad y variedad de teorías sobre el desarrollo que emergen después de la crisis global de los años 30, conocida como la gran recesión económica, y que se acentúan en la década del 40 después de la II guerra mundial. De estas teorías no todas se configuran como paradigmas².

La noción que toma mayor fuerza en ese momento es la de desarrollo como crecimiento económico (Casella, 2008); este planteamiento, que surgió por parte de los economistas, sostiene que para generar mayor nivel de bienestar en las sociedades se requiere de tasas de crecimiento económico sostenidas en el tiempo.

2 "Según Thomas Kuhn un paradigma es una configuración de supuestos vinculados que constituyen una constelación de creencias y valores compartidos por los miembros de una comunidad que puede ser empleada como modelo o sistema para resolver problemas" (Gutiérrez-Garza y González-Gaudiano, 2010, p. 183) 
Este paradigma se impuso a lo largo del tiempo siendo precepto para la toma de decisiones políticas, principalmente promovidas por los países de mayor riqueza y poder sobre los países de menor desarrollo económico, mediados por los organismos multilaterales creados desde la década de los 40 como la ONU y la OMS; en América, la Comisión Económica para América Latina y el Caribe (CEPAL), el Banco Mundial, entre otros (Casella, 2008; De Souza-Silva, 2010; Siero et al., 2015).

Como el objetivo de este estudio es aproximarse a la relación desarrollo y salud, resulta complejo abordar específicamente la totalidad de las teorías, de ahí que se requiere agruparlas con algún criterio objetivo según sus características más relevantes.

De esta manera, se establecen tres categorías que se diferencian por criterios de tiempo, enfoque, y trascendencia. La primera corresponde a Desarrollo 1.0, en la que se incluyen todas las teorías y modelos que participan en el desarrollo y cuyo común denominador es el privilegio del crecimiento económico (Manet, 2013; Siero et al., 2015), del nivel de riqueza, y la subvaloración de los ecosistemas y del bienestar social, físico y mental de los individuos y colectivos.

Con altibajos, este modelo ha imperado y ha sido administrado o complementado por teorías economicistas que han motivado a buscar mayores niveles de riqueza para los países mediante estrategias como la globalización de los mercados y la adopción de modelos neoliberales de gobernanza en los países de menor desarrollo.

La literatura analizada en esta revisión nos muestra algunas evidencias de los efectos que este modelo ha tenido para la sociedad y para el planeta. A pesar de que las investigaciones de Brenner evidencian los daños para la salud en los momentos de crisis o decrecimiento económico y sus contradictores muestren lo contrario, no es posible ser concluyente, dado que estos debates no solo se centran en las metodologías, sino que existen intereses y visiones divergentes en temas sociales y de salud (García, 2017; Brenner, 2005).

No obstante, en el ámbito de la salud física y mental, emergen con aumento preocupante las llamadas Enfermedades No Transmisibles (ENT), que se están convirtiendo en la principal causa de morbi-mortalidad y de externalidades globales, muchas de las cuales se asocian a través de la evidencia a factores ambientales derivados de la acelerada urbanización, consumos desenfrenados, carencias en las dotaciones de saneamiento básico, aumento de la contaminación en el suelo, agua y aire, en general a lo que la OMS ha reconocido como los DSS (GBD 2015 Neurological Disorders Collaborator Group, 2017; GBD 2015 Risk Factors Collaborators, 2016; Mora-Alvarado y Portuguez Barquero, 2009; Rubio-Guerrero y Fierro-Celis, 2015; Tafani et al., 2015). 
Los estudios seleccionados evidencian, además, otros impactos negativos del modelo, entre los que sobresalen: incontrolables tasas de pobreza material y económica, altas tasas de desempleo y precarización del mismo, conflictos sociales y políticos, desigualdades socio económicas, tasas de morbi-mortalidad prevenible elevadas, inequidades en cuanto a la distribución de las riquezas generadas, exacerbación de los problemas ambientales por depredación de ecosistemas y el cambio climático (Rubio-Guerrero y Fierro-Celis, 2015).

Como respuesta a lo anterior, desde el modelo de Desarrollo 1.0 se han hecho esfuerzos globales por mitigar los problemas sociales y ambientales adjudicados a este; es así como se han formulado teorías e intervenciones centradas en: disminución de las desigualdades, aumento de la equidad social, mejoramiento de la salud pública, disminución de la pobreza económica y sostenibilidad ambiental (Arias, 2017; GarcíaRodríguez et al., 2017; Lustig, 2007; Muñoz-Muñoz, 2011; Pratt \& Loff, 2012; SalaverryGarcía, 2013; Sarmiento-Suárez, 2017; Zurbriggen y Travieso, 2016).

Aparecen en este marco conceptos como capital social, capital humano, desarrollo humano, desarrollo social, responsabilidad social, emprendimiento social, innovación social, mercadeo social, gerencia social, entre otros. Estos nuevos conceptos priorizan el bienestar de los seres humanos, de los colectivos y de los ecosistemas, de ahí su denominación de Desarrollo 2.0, debido a que, aunque representan un avance significativo, no han logrado posicionarse como paradigmas de desarrollo y no desplazan la noción del Desarrollo 1.0, supeditando todos los esfuerzos al mejoramiento económico y tecnológico (Grabois-Gadelha et al., 2012). En esta categoría se pueden articular todas aquellas nociones, teorías o modelos alternos que no logran desprenderse del crecimiento de riquezas como fin humano y social.

Todos los esfuerzos que realizan los organismos multilaterales por mejorar los niveles de bienestar y la sostenibilidad en el planeta como los Objetivos de Desarrollo Sostenible (ODS), antes los Objetivos de Desarrollo del Milenio, acuerdos multilaterales como el de Rio, el de Kioto, el de París, por ejemplo, en asuntos de cambio climático y sostenibilidad (Urbina-Fuentes, Jasso-Gutierrez, Schiavon-Ermani, Lozano y Finkelman, 2017), podrían clasificarse en este Desarrollo 2.0, pues, si bien formulan objetivos para que los gobiernos mejoren o mitiguen las condiciones de vida de sus ciudadanos y eviten la tragedia ambiental, las condiciones estructurales del paradigma de desarrollo imperante no se tocan (Tellería, 2015).

En la categoría Desarrollo 3.0, se pueden agrupar todas aquellas teorías o nociones que desde la crítica a los demás modelos, plantean una alternativa opuesta, en la que ni la riqueza material ni el bienestar constituyen el objetivo del desarrollo (Escobar y Esteva, 2017). Se habla, por ejemplo, de otros aspectos como las capacidades, la educación y la libertad (Cejudo-Córdoba, 2006), y, si bien siguen utilizando este mismo vocablo 
(desarrollo), su esencia es el ser humano en relación con sus entornos, siendo un componente más de los ecosistemas en que habita (St'ahel, 2019). En este modelo se agregan otras dimensiones a la noción del desarrollo economicista, como la social, la ambiental, la cultural y hasta la espiritual.

Para (Gamarra-Pérez, 2015), es necesario un cambio axiológico en el pensamiento humano articulado con sus valores, la naturaleza y su espiritualidad si se desea un cambio real en las políticas de desarrollo. En esta perspectiva, la visión del desarrollo se construye desde el holismo, desde las subjetividades, que una vez sean apropiadas por las comunidades y mediante procesos democráticos pueden llegar a ser políticas estatales con alcance global.

En la lectura transversal de estos tres modelos, el desarrollo 3.0 es realmente el considerado modelo alternativo, incluso algunos autores como (Gutiérrez-Garza y González-Gaudiano, 2010) al analizar las bases de un modelo de desarrollo sustentable, considera este concepto como un "oxímoron" pues ambas palabras son mutuamente excluyentes, es decir en el modelo de desarrollo alternativo se contempla la necesidad de un nuevo discurso, ya que el desgaste y manipulación del vocablo "desarrollo" ya no brinda la posibilidad de defender su idea implícita y por más que se le agreguen adjetivos para maquillarlo siempre conserva su sesgo original (De Souza-Silva, 2010).

En América Latina, se podría decir que se han dado intentos de instaurar en algunos países el modelo de Desarrollo 3.0 incorporando la noción de Postdesarrollo.

Los planes de desarrollo en Ecuador y Bolivia, incluyeron la noción del Buen Vivir que reemplaza al concepto de desarrollo (Unceta-Satrustegui, 2013). No obstante, (Sager, 2014) afirma que en el caso boliviano se identifican contradicciones entre el discurso del buen vivir y la sostenibilidad con las políticas reales, esto debido a la ausencia de un consenso en torno al modelo adecuado generado por la dependencia económica de Bolivia en la extracción de los recursos no renovables y la relevancia de ciertos intereses sociales contradictorios. Por otra parte, en algunos países europeos, inicialmente en Francia y luego en España e Italia se habla de la noción de Decrecimiento; ambas nociones, la Andina y la Europea, contenidas en la noción de postdesarrollo, rechazan la idea hegemónica del bienestar soportada en el crecimiento económico (Unceta-Satrustegui, 2013).

Estos tres modelos de desarrollo (1.0, 2.0 y 3.0) se presentan de forma simultánea y son interpretados por los actores políticos de cada territorio para la formulación e implementación de políticas, de tal forma que se asigna a cada uno rasgos ideológicos, antropológicos, filosóficos y políticos que generan grandes polarizaciones en la opinión de los ciudadanos, afincando su influencia a través de los diferentes medios y estrategias de información parcializados según sus intereses específicos. 
Sánchez-Ordóñez

Es evidente la supremacía del Desarrollo 1.0 en el concierto global, principalmente en aquellos países donde impera el modelo de gobernanza neoliberal. El Desarrollo 2.0 está presente en aquellos países que sostienen aún el llamado Estado de bienestar, en los que, a pesar de las complejas situaciones que impone la globalización, la transición demográfica, los movimientos poblacionales y el cambio climático, sostienen políticas redistributivas para sus ciudadanos mediante una gran batería de servicios y programas de inclusión, asistencia, equidad e igualdad (Lustig, 2007; Pratt \& Loff, 2012; Salaverry-García, 2013) que garantizan un nivel de vida alto para la mayoría de las personas, no obstante los desafíos económicos que este modelo requiere.

Para los países que han optado por el Desarrollo 3.0, la situación es más compleja, dado que el nivel de riqueza y, en la mayoría de los casos, problemáticas como corrupción, inestabilidad política de su modelo de gobernanza, presiones transnacionales y necesidades de sus ciudadanos que son influidos por la globalidad, impiden la consolidación real de un modelo alternativo.

Comunidades indígenas, afrodescendientes o campesinas, entre otras, han hecho esfuerzos de resistencia contra las tendencias globales de desarrollo, defendiendo conceptos como el buen vivir, planes de vida, florecimiento humano, etno desarrollo, entre otros, asociados al Desarrollo 3.0 (Escobar, 2007).

\subsection{La salud y el desarrollo}

La salud, en tanto bien público, constituye una de las principales responsabilidades del Estado moderno. Siendo esta entendida como un medio para el bienestar de las personas, toda inversión que se realice desde el Estado o desde otros sectores implicados reditúa en capital humano y productividad; la salud es, además, una capacidad básica para el desarrollo humano y el bienestar social (Agudelo, 1999; García-Rodríguez et al., 2017).

Así mismo, se considera que la inversión en salud provoca importantes externalidades positivas, dado que en el caso, por ejemplo, de los programas de prevención, promoción y rehabilitación de la salud, éstos apuntan a beneficiar a toda la comunidad. El retorno calculado de esta inversión, además de ser individual y colectivo para los ciudadanos, también es evidente en el aumento de la productividad y por tanto de la economía en su conjunto, representando mayor crecimiento económico para los países. Salud y desarrollo son entendidos como partes de un mismo proceso dinámico, que tiene al ser humano, el bienestar y la equidad como sus ejes centrales (Muñoz-Muñoz, 2011).

Todas las intervenciones que se agencian por parte de los Estados, desde las políticas públicas hasta los proyectos puntuales enmarcados dentro del anhelo de desarrollo afectan estructural e integralmente a las sociedades. Igualmente, se ha hecho más visible la relación 
entre aspectos estructurales de las sociedades y los niveles de salud, reafirmando cada vez la vinculación entre el desarrollo y la salud (Ríos-Osorio, Salas-Zapata \& Ortiz-Lobato, 2012).

Independientemente del modelo de desarrollo en el que nos ubiquemos, la relación entre salud y bienestar mediada por el contexto produce características diferentes en cada una de las dimensiones de la salud. La evidencia científica ha demostrado cómo los efectos del desarrollo 1.0 se manifiestan en el alto número de ENT asociadas a estilos de vida marcados por los tipos de dieta, calidad de los alimentos, ritmos acelerados de trabajo, exposición a altos niveles de contaminantes ambientales (World Health Organization, 2016).

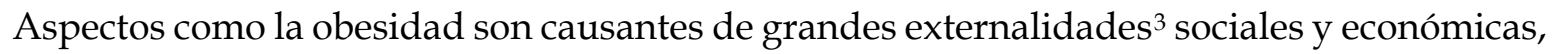
igual ocurre con un gran número de enfermedades relacionadas directamente con los DSS propios de cada contexto.

Las ENT representan cada vez más una carga muy costosa para los países de bajos y medianos ingresos, lo que significa grandes externalidades negativas para los Estados y las familias. Solo uno de los principales factores de riesgo, el consumo de tabaco, impone casi 6 millones de vidas anualmente y cuesta entre 1 y $2 \%$ del PIB global cada año (Goodchild, Perucic \& Nargis, 2016; Organización Mundial de la Salud (OMS), 2018).

El impacto que están generando las ENT para el desarrollo en todos los países ha ocupado la mayor atención por parte de todos los organismos multilaterales como se evidencia en sendas declaraciones de la ONU desde el 2012 (Buss, Fonseca, Galvão, Fortune y Cook, 2016).

Lamentablemente, muchos compromisos políticos no se están cumpliendo, las medidas nacionales contra las ENT son, en el mejor de los casos, desiguales. Las inversiones nacionales siguen siendo mínimas, y no están fluyendo fondos suficientes a nivel internacional. Se han formulado numerosas políticas, pero las estructuras y los recursos para implementarlas son insuficientes, a pesar de contar con evidencia científica para la acción (OMS, 2018).

Aparte de las enfermedades físicas, los trastornos mentales suponen una enorme carga de morbilidad en las sociedades (Sarmiento-Suárez, 2017). La depresión es la principal causa de discapacidad en todo el mundo y cada año se suicidan casi 800.000 personas; los trastornos mentales graves disminuyen la esperanza de vida en 10 a 20 años, debido principalmente a ENT no tratadas.

3 Las externalidades hacen referencia a aquellos resultados que pueden traer beneficios (positivas) o causar daños diversos (negativas) no esperados por efectos de una intervención sea Estatal, Organizacional o de grupos sociales.

Prospectiva. Revista de Trabajo Social e intervención social • No. $31 ・$ ene.jun. $2021 \bullet$ pp. 413-436 e-ISSN: 2389-993X • https://doi.org/10.25100/prts.v0i31.8934 
Sánchez-Ordóñez

Según el informe de la comisión de la OMS de 2018, los principales obstáculos para la mejor intervención de las ENT por parte de los países son: falta de voluntad política, compromiso, capacidad y medidas; falta de políticas y planes concernientes a ENT; dificultades para el establecimiento de prioridades; repercusiones de factores económicos, comerciales y de mercado; capacidad técnica y operacional insuficiente; financiación nacional e internacional insuficiente para intensificar las respuestas nacionales a las ENT; y falta de rendición de cuentas (OMS, 2018).

Las recomendaciones de la OMS son pertinentes cuando habla de volcar las acciones a la prevención y a la promoción de los DSS, y cuando recomienda incorporar la salud en todas las políticas (Alleyne, 2015) llamando a la intersectorialidad e interdisciplinariedad; sin embargo, profundizando en las características de los DSS, nos encontramos con condiciones, situaciones o fenómenos complejos y estructurales, de los cuales muchos rebasan el enfoque de promoción.

La relevancia del desarrollo 1.0 obliga a que las intervenciones agenciadas involucren solo a consumidores objetivo, pero no ataca la amplia gama de factores interconectados como es el caso de la producción de alimentos, el procesamiento y su distribución. De hecho, estos factores pueden afectar el consumo nutricional y es importante incluirlos para mejorar la dieta.

Forouzanfar et al. (2016) plantean que los malos hábitos alimenticios se asocian con una variedad de enfermedades crónicas y pueden potencialmente ser un importante contribuyente a la mortalidad por ENT en todos los países del mundo. Por ello, se destaca la necesidad urgente de esfuerzos globales coordinados para mejorar la calidad de la dieta humana, mediante la implicación de todos los actores globales del sistema alimentario.

\subsection{Desafíos para el desarrollo y la salud en el siglo XXI}

A pesar de que la literatura revisada demuestra avances en el desarrollo económico en la mayoría de los países de la muestra, es evidente la brecha existente entre los países de mayor riqueza y los de menor desarrollo económico. También se puede observar que el aumento en la expectativa de vida ha sido significativo, aunque desigual también durante estas décadas; esto debido a varios aspectos, principalmente al avance en las tecnologías, los conocimientos en salud física, a los nuevos tratamientos y medicamentos que han permitido superar enfermedades antes mortales.

Si bien es cierto que una comparación estadística entre los países con indicadores económicos e indicadores sociales basados en los datos oficiales, no permite inferir con precisión estadística lo que sucede dado que hay muchos elementos de distorsión que impiden estas comparaciones, sí nos ayuda a reflexionar sobre la relación desarrollo-salud- 
bienestar, sobre todo si se parte de los postulados del desarrollo; es decir, al hacer estas comparaciones hipotéticamente se esperaría encontrar que los países de mayor riqueza económica, y especialmente aquellos en los que la riqueza promedio de la población es más alta, los niveles de calidad de vida, bienestar y salud deben ser los más altos (MezaCarvajalino, 2006).

En este sentido resulta relevante el caso del continente americano, en el que los mayores referentes de desarrollo son los Estados Unidos y Canadá, esto por ser los que más alto nivel de PIB producen según la medición del Banco Mundial. Sin embargo, son muy dicientes los datos de países pequeños como Cuba y Costa Rica, que, a pesar de tener menores niveles de riqueza económica, tienen mayor esperanza de vida que Estados Unidos. El caso cubano es de analizar en detalle (Sanabria-Ferrand y Pino-Robledo, 2018), pues su PIB per cápita es menos de la mitad del que tiene Costa Rica, con grandes dificultades económicas producto del bloqueo comercial y financiero del que es objeto por parte de Estados Unidos desde hace años, lo que le cierra opciones importantes de crecimiento en el mercado internacional.

Se ratifica con estos datos del continente americano la contradicción del paradigma del desarrollo 1.0 o hegemónico, debido a que es evidente que los mayores niveles de salud y bienestar de un país no están condicionados por el nivel de riqueza de sus ciudadanos.

Otro aspecto significativo que surge en este estudio y que podría ser una paradoja más del desarrollo, es que, en su mayoría, los países más saludables y de mayor riqueza presentan los índices más altos en cuanto esperanza de vida; sin embargo, tienen los índices más bajos de población joven (a excepción de Israel), las tasas más altas de población mayor de 65 años (a excepción de Israel) y las tasas más bajas de fecundidad global (a excepción de Israel). Lo anterior nos indica a priori que, al mejorar sus niveles de desarrollo y salud, los países también enfrentan un gran reto de sostenibilidad demográfica en el futuro cercano, dado el envejecimiento de su población, de la baja en el número de niños que nacen y de la disminución de su población joven.

El caso de Israel es llamativo ya que, teniendo un buen nivel de riqueza, muestra tasas altas de fecundidad, de población joven, contrastadas con bajas tasas de población mayor de 65 años. Por ello será de gran aporte para el mundo conocer en detalle las acciones que este país adelanta para armonizar la ecuación desarrollo y salud sin poner en riesgo su sostenibilidad demográfica futura. 


\section{Conclusiones}

Salud y desarrollo como discursos globales comparten sus orígenes en el contexto de la segunda posguerra mundial y, a pesar de que cada uno toma su rumbo diferenciado en la teoría, no es así en la práctica, en la que se muestran unidos.

Actualmente ambos conceptos han sido superados por las realidades contemporáneas, hoy se reconocen la multidimensionalidad y gran articulación que tienen con aspectos sociales, políticos, culturales, ambientales y espirituales, conformando una parte indisoluble e imprescindible del bienestar humano, la calidad de vida o la felicidad.

Los retos que impone el siglo XXI acrecientan el debate entre desarrollo y salud y ponen al descubierto los factores realmente relevantes para intervenir los DSS, dejando visibles los aspectos éticos y políticos de los gobiernos, principalmente en los países de alto desarrollo que por su nivel de riqueza económica, tecnológica y cultural son los llamados a mostrar las sendas correctas para que transiten los países de menor desarrollo.

El desarrollo 1.0, a pesar de generar avances en el crecimiento de la riqueza de los países y de mejorar algunas condiciones para el alargue de la vida de las personas, ha demostrado impactos negativos sobre la salud humana y la sostenibilidad ecológica y social del planeta. Avanzar al desarrollo 2.0, como se viene planteando globalmente al complementar el desarrollo con categorías como sostenible o sustentable, humano, social, si bien es cierto acrecienta el anhelo de los pueblos, no satisface integralmente los retos que se enfrentan pues siguen siendo el crecimiento económico y la riqueza material el fundamento.

El desarrollo 3.0 por su naturaleza holística y multidimensional constituye una alternativa total a los paradigmas de desarrollo $\mathrm{y}$, aunque desde la perspectiva de la intervención son pocas las evidencias disponibles, el tamaño de los desafíos globales amerita explorar este camino con mayor decisión, lo que obliga a dejar de ver la salud y el desarrollo como fines en sí mismos. Esta noción de desarrollo impone cambios estructurales $\mathrm{y}$, sobre todo, un gran cambio cultural que será necesario abordar de cara a la sostenibilidad del planeta para las generaciones futuras.

Con esta revisión queda planteada la necesidad de generar evidencias más contundentes sobre la relación desarrollo y salud, así como la urgencia de incorporar nociones más amplias como la del desarrollo 3.0 a las intervenciones que se proponen desde las políticas, los programas y proyectos de los países y, en general, las organizaciones de todo tipo, pues el proceso de cambio es lento pero obligatorio.

Los hallazgos de esta revisión ponen en cuestión los discursos del desarrollo sobre los que se han forjado las políticas de cada uno de los países, y nos obligan a profundizar el análisis en aras de aportar nuevos paradigmas que de una vez por todas cierren las 
mencionadas brechas existentes entre desarrollo, salud y bienestar e iluminen la formulación e implementación de intervenciones que garanticen una mejor vida para todos los seres humanos y sus respectivos entornos.

\section{Referencias bibliográficas}

Agudelo, C. (1999). Desarrollo y Salud. Revista de Salud Pública, 1(1), 17-28.

Alleyne, G. A. O. (2015). Salud en todas las políticas: El control y la prevención de enfermedades crónicas no transmisibles. Salud Publica de Mexico, 57(3), 284-287.

Álvarez-Castaño, L. S. (2014). Los determinantes sociales y económicos de la salud. Asuntos teóricos y metodológicos implicados en el análisis. Gerencia y Políticas de Salud, 13(27), 28-40. Doi: 10.11144/Javeriana.rgyps13-27.dses.

Arias, J. (2017). La sostenibilidad justa como paradigma sistémico ambiental. Gestión y Ambiente, 20(2), 232-243.

Brenner, M. H. (2005). Commentary: Economic growth is the basis of mortality rate decline in the 20th century - Experience of the United States 1901-2000. International Journal of Epidemiology, 34(6), 1214-1221. Doi: 10.1093/ije/dyi146.

Buss, P. M., Fonseca, L. E., Galvão, L. A., Fortune, K., y Cook, C. (2016). health in all policies the parthnership for sustainable development. Revista Panamericana de Salud Publica, 40(3), 186-191.

Casella, A. (2008). Teorías latinoamericanas del desarrollo: el estado sustituto del individuo. Espacio Abierto, 17(3), 475-497.

Cejudo-Córdoba, R. (2006). Dessarrollo humano y capacidades. Aplicaciones de la teoría de las capacidades de Amartya Sen a la educación. Revista Española de Pedagogia, 64(234), 365-380.

De Souza-Silva, J. (2010). Otro paradigma para el desarrollo humano sustentable. Ascenso y declinación de la "idea de desarrollo". Textual, enero, (55), 9-26.

Escobar, A. (2007). La invención del Tercer Mundo: construcción y deconstrucción del desarrollo. Caracas, Venezuela: Fundación Editorial el perro y la rana.

Escobar, A., y Esteva, G. (2017). Postdesarrollo a los 25: sobre 'estar estancado' y avanzar hacia adelante, hacia los lados, hacia atrás y de otras maneras. Polisemia, 12(22), 17-32. Doi: 10.26620/uniminuto.polisemia.12.22.2016.17-32.

Feigin, V. L., Krishnamurthi, R. V., Theadom, A. M., Abajobir, A. A., Mishra, S. R., Ahmed, M. B., ... Zaki, M. E. (2017). Global, regional, and national burden of neurological disorders during 1990-2015: a systematic analysis for the Global Burden of Disease Study 2015. The Lancet Neurology, 16(11), 877-897. Doi: 10.1016/S1474-4422(17)302995.

Forouzanfar, M. H., Afshin, A., Alexander, L. T., Biryukov, S., Brauer, M., Cercy, K., ... Zhu, J. (2016). Global, regional, and national comparative risk assessment of 79 behavioural, environmental and occupational, and metabolic risks or clusters of risks, 1990-2015: a 
systematic analysis for the Global Burden of Disease Study 2015. The Lancet, 388(10053), 1659-1724. Doi: 10.1016/S0140-6736(16)31679-8.

Gamarra-Pérez, R. A. (2015). Algunas consideraciones al modelo de desarrollo capitalista vigente en américa Latina. FIDES ET RATIO, 9, 81-96.

García, M. (2017). Crisis económicas y salud en controversia: el “debate Brenner”, 1970-2012. Revista Ciencias de la Salud, 15(1), 87-104.

García-Rodríguez, J. F., García-Fariñas, A., Priego-Hernández, O., y Martíez-Pérez, L. (2017). Salud desde una perspectiva económica. Importancia de la salud para el crecimiento económico, bienestar social y desarrollo humano. Salud en Tabasco, 23(1-2), 44-47.

GBD 2015 Neurological Disorders Collaborator Group. (2017). Global, regional, and national burden of neurological disorders during 1990-2015: a systematic analysis for the Global Burden of Disease Study 2015. The Lancet Neurology, 16(11), 877-897. Doi: 10.1016/S1474-4422(17)30299-5.

GBD 2015 Risk Factors Collaborators (2016). Global, regional, and national comparative risk assessment of 79 behavioural, environmental and occupational, and metabolic risks or clusters of risks, 1990-2015: a systematic analysis for the Global Burden of Disease Study 2015. The Lancet, 388(10053), 1659-1724. Doi: 10.1016/S0140-6736(16)31679-8.

Goodchild, M., Perucic, A., \& Nargis, N. (2016). Modelling the impact of raising tobacco taxes on public health and finance. Bull World Health Organ, 94(4), 250-257.

Grabois-Gadelha, C. A., Costa, L. S., \& Maldonado, J. (2012). O Complexo EconômicoIndustrial da Saúde e a dimensão social e econômica do desenvolvimento. Revista de Saúde Pública, 46(supl.1), 21-29.

Gutiérrez-Garza, E., y González-Gaudiano, E. (2010). De las teorías del desarrollo al desarrollo sustentable. Nuevo León, México: Universidad Autónoma de Nuevo León.

Higuita-Gutiérrez, L. F., y Cardona-Arias, J. A. (2018). Índice de desarrollo humano y eventos de salud pública: revisión sistemática de la literatura 1990-2015. Revista Facultad Nacional de Salud Pública, 36(1), 5-16. Doi: 10.17533/udea.rfnsp.v36n1a02.

Lustig, N. (2007). Salud y desarrollo economico: el caso de Mèxico. El Trimestre Economico, 74(296(4)), 793-822.

Mamédio, C., Roberto, M., \& Nobre, C. (2007). the Pico Strategy for the Research Question. Revista Latino-Am Enfermeria, 15(3), 1-4. Doi: 10.1590/S0104-11692007000300023.

Manet, L. (2013). Modelos de desarrollo regional : teorías y factores determinantes. Noesis. Revista de Ciencias Sociales y Humanidades, 23(46), 18-56.

Meza-Carvajalino, C. A. (2006). Las reformas estructurales de los años 90 en América Latina y sus efectos en el servicio de salud y la calidad de vida. Equidad y Desarrollo, (5), 4760. Doi: 10.19052/ed.345.

Moher, D., Liberati, A., Tetzlaff, J., Altmane, D. G, y Grupo PRISMA. (2014). Ítems de referencia para publicar Revisiones Sistemáticas y Metaanálisis: La Declaración PRISMA. Revista Española de Nutricion Humana y Dietetica, 18(3), 172-181. 
Sánchez-Ordóñez

Mora-Alvarado, D. A., y Portuguez-Barquero, C. F. (2009). Salud y desarrollo en el mundo: emisiones de $\mathrm{CO}^{2}$ versus los indicadores de saneamiento y educaciòn. Revista Costarricense de Salud Pùblica, 18(2), 79-83.

Muñoz-Muñoz, S. (2011). Salud y desarrollo en el contexto latinoamericano. Revista Cubana de Salud Publica, 37(2), 167-175.

Organización Mundial de la Salud. (1946). Constitución de la Organización Mundial de la Salud. Nueva York: OMS.

Organización Mundial de la Salud. (2018). Es hora de actuar: Informe de la Comisión Independiente de al to nivel de la OMS sobre Enfermedades no Transmisibles. Ginebra, Suiza: Organización Mundial de la Salud.

Organización Panamericana de la Salud. (2018). Indicadores básicos 2018. Situación de Salud en las Américas. Washington, USA: OPS/OMS.

Pratt, B., \& Loff, B. (2012). Health research systems: promoting health equity or economic competitiveness? Bulletin of the World Health Organization, 90(1), 55-62. Doi: 10.2471/BLT.11.092007.

Programa de las Naciones Unidas para el Desarrollo. (2019). Panorama general. Informe sobre Desarrollo Humano 2019: Más allá del ingreso, más allá de los promedios, más allá del presente: Desigualdades del desarrollo humano en el siglo XXI. Nueva York: Programa de las Naciones Unidas para el Desarrollo. Recuperado de http://hdr.undp.org/sites/default/files/hdr_2019_overview_-_spanish.pdf.

Ríos-Osorio, L. A., Salas-Zapata, W. A., \& Ortiz-Lobato, M. (2012). Concepts Associated with Health from the Perspective of Sustainable Development. Saúde e Sociedade, 21(3), 735746.

Rubio-Guerrero, G., y Fierro-Celis, F. A. (2015). Desarrollo sostenible y mercados eficientes: hacia la construcción de un modelo teórico. Contexto, 4, 71-80.

Ruiz-Lurduy, R., Rocha-Buelvas, A., Pérez-Hernández, E. y Córdoba-Sánchez, C. (2016). Desarrollo social y salud pública. Reflexiones en torno a la interculturalidad. Revista Facultad Nacional de Salud Pública, 34(3), 380-388.

Sager, F. (2014). Bolivia entre el Desarrollo Sostenible y la Explotación de la Naturaleza - El marco político y jurídico de los Sistemas Agroforestales como ejemplo para una agricultura sostenible. Acta Nova, 6(3), 194-209.

Salaverry-García, O. (2013). La Inequidad en Salud. Su Desarrollo Histórico. Revista Peruana de Medicina Experimental y Salud Publica, 30(4), 709-714.

Sanabria-Ferrand, P. A., y Pino-Robledo, S. (2017). Relaciones entre la presencia de evitación experiencial y el nivel de actividad física de jóvenes universitarios. Revista de Psicología del Deporte, 26(2), 119-122.

Sarmiento-Suárez, M. J. (2017). Salud Mental y desarrollo. Revista Medicina, 39(3), 246-254.

Sen, A. (2000). EL desarrollo como libertad. Gaceta Ecológica, (55), 14-20.

Sen, A. (2004). ¿Como importa la cultura en el desarrollo? Letras Libres, 6(71), 23-30. 
Sánchez-Ordóñez

Siero, C., Rodríguez, R., y Hernández, S. (2015). ¿Ha sido importante la cooperación internacional para el desarrollo y el crecimiento económico? Una evidencia de datos panel para Nicaragua y Centroamérica. Encuentro, (102), 52-74.

St'ahel, R. (2019). Sustainable development in the shadow of climate change. Civitas - Revista de Ciências Sociais, 19(2), 337-353.

Tafani, R., Chiesa, G., Caminati, R., y Gaspio, N. (2015). Desarrollo, medio ambiente y salud. Revista de Salud Pública, 19(1), 22-37.

Tellería, J. (2015). ¿Seguimos hablando de desarrollo? El paradigma del desarrollo humano del PNUD como saber-poder. Nómadas, (43), 241-251.

Unceta-Satrustegui, K. (2013). Decrecimiento y Buen Vivir iparadigmas convergentes? debates sobre el postdesarrollo en Europa y América Latina. Revista de Economía Mundial, (35), 197-2016.

Urbina-Fuentes, M., Jasso-Gutiérrez, L., Schiavon-Ermani, R., Lozano, R., y Finkelman, J. (2017). La transición de los objetivos de desarrollo del milenio a los objetivos de desarrollo sostenible desde la perspectiva de los determinantes sociales de la salud y la equidad en salud. Gaceta Medica de Mexico, 153, 697-730.

World Health Organization. (2016). Ambient air pollution: a global assessment of exposure and burden of disease. Geneve, Switzerland: World Health Organization.

Zurbriggen, C., y Travieso, E. (2016). Hacia un nuevo Estado desarrollista: Desafíos para América Latina. Perfiles Latinoamericanos, 24(47), 259-281. Doi: 10.18504/pl2447-0042016. 


\section{OTROS ARTÍCULOS DE PROSPECTIVA No. 31 DE 2021}

\section{PRESENTACIÓN}

Presentación. Reflexiones sobre desafios al publicar sistematizaciones

Rosa María Cifuentes-Gil

\section{EDITORIAL}

Reflexiones sobre Trabajo Social: aportes de la Sistematización

María Rocío Cifuentes-Patiño

\section{ARTÍCULOS}

Hacer lo que se sabe, pensar lo que se hace. La sistematización como modalidad investigativa Alfonso Torres-Carrillo

Aportes y desafios de la Sistematización de experiencias en el Trabajo Social y la extensión crítica. Apuntes y reflexiones desde la perspectiva de la Educación Popular

María Rosa Goldar

Valeria Chiavetta

La sistematización en Trabajo Social y la epistemología feminista del punto de vista. Diálogos sobre la producción de conocimiento sustentada en experiencias

Ruth Noemí Parola

María Florencia Linardelli

La Sistematización investigativa de las experiencias: del baile de los que sobran a la fiesta de los que faltan

María Belén Ortega-Senet

Sistematización y Trabajo Social en Chile. El largo y sinuoso camino

Patricia Lorena Castañeda-Meneses

Ana María Salamé-Coulon
Sentipensar la pandemia COVID-19 desde la sistematización de la experiencia en Trabajo Social: reflexiones del profesor Oscar Jara Holliday

Elia Sepúlveda-Hernández

La sistematización de experiencias, una investigación social cualitativa que potencia buenas prácticas de convivencia y gobierno. La experiencia de un conjunto residencial multifamiliar en Cali, Colombia Martha Lucia Echeverry-Velásquez Manuela Prada-Dávila

Construcción de subjetividades epistemológicaspolíticas de profesoras y profesores de Investigación social en una universidad privada y confesional en Bogotá

Giovanni Mora-Lemus

Sistematización de la experiencia Reconocimiento de los derechos humanos del adulto mayor en dos familias residentes en Cali y Valledupar (Colombia)

Lina María Cuello-Lacouture

Jimena del Pilar Jaramillo-Jaramillo

La memoria transformadora como estrategia de intervención profesional en los procesos de reconciliación social: comprensión a partir de mujeres campesinas, excombatientes y jóvenes en Manizales, Colombia

Yeimmy Stephania Corredor-Sotelo

Juliana Fuertes-Fuertes

Sistematización de una estrategia de educación informal implementada en personas privadas de la libertad en el establecimiento penitenciario de mediana seguridad y carcelario de Barranquilla, Colombia

Rafael Humberto Herrera-Mercado Rafael Alberto Zambrano-Vanegas 
Aportes significativos del proceso de intervención comunitaria con la Escuela Popular de Comunicación Alternativa Jaime Garzón de la ciudad de Cúcuta, Colombia

Carlos Lasso-Urbano

La sistematización de la intervención como metodología de investigación en Trabajo Social. Importancia práctica y teórica de la fase de recogida de datos en la intervención social según experiencia del Programa de Apoyo a las Familias en Zaragoza, España

Elisa Esteban-Carbonell

Nuria Del Olmo-Vicén

Papel de la sistematización de experiencias en los procesos de evaluación de intervenciones de salud pública en la Comuna Saludable por la Paz, Cali - Colombia

Jenny Faisury Peña-Varón

Paola Andrea Marín-Velásquez

Janeth Mosquera-Becerra

Experiencia de intervención social en hogares comunitarios integrales del barrio Alfonso Bonilla Aragón, Cali - Colombia

Julián Alexander Montaño-Cárdenas

Las políticas sociales y el gobierno de la "población indígena". Estrategias y regulaciones en el multiculturalismo chileno

Rodrigo Agustín Navarrete-Saavedra

Representaciones sociales sobre estilo de autoridad y tipos de interacción en cuidadores de residencias de protección infantil en Chile

Marcelo Gallegos-Fuentes
Carmen Gloria Jarpa-Arriagada

Reflexiones sobre inseguridad social y cuestiones penales. Una respuesta estratégica a partir de experiencias de cooperativismo con ex detenidos en Argentina

Analia Elizabeth Otero

Yael Yanina Barrera

Desarrollo y salud: la emergencia de un nuevo paradigma

Jesús María Sánchez-Ordóñez

Trabajo Social en ejercicio libre: la perspectiva profesional en España

Paula Frieiro-Padín

Tamara Fernández-Arias

Rubén González-Rodríguez

\section{RESEÑAS}

Social Work and the City: Urban Themes in 21stCentury Social Work

Felipe Saravia-Cortés

Respuestas del Trabajo Social ante emergencias sociales y problemáticas sociales complejas de México y España

Felipe Saravia-Cortés

El feminismo, el género y la profesionalización del trabajo social en Colombia (1936-2004)

Ambar Oriana Serna-Lombo

El puño invisible. Arte, revolución y un siglo de cambios culturales

Carlos Arturo Robledo-Marín

\section{PROSPECTIVA}

No. $31 \bullet$ ene.-jun. 2021

e-ISSN: 2389-993X • Universidad del Valle 